\title{
Default mode network connectivity encodes clinical pain: An arterial spin labeling study
}

\section{Citation}

Loggia, Marco L., Jieun Kim, Randy L. Gollub, Mark G. Vangel, Irving Kirsch, Jian Kong, Ajay D. Wasan, and Vitaly Napadow. 2013. “Default Mode Network Connectivity Encodes Clinical Pain: An Arterial Spin Labeling Study." Pain 154 (1) (January): 24-33. doi:10.1016/j.pain.2012.07.029.

\section{Published Version}

doi:10.1016/j.pain.2012.07.029

\section{Permanent link}

http://nrs.harvard.edu/urn-3:HUL.InstRepos:36303921

\section{Terms of Use}

This article was downloaded from Harvard University's DASH repository, and is made available under the terms and conditions applicable to Other Posted Material, as set forth at http:// nrs.harvard.edu/urn-3:HUL.InstRepos:dash.current.terms-of-use\#LAA

\section{Share Your Story}

The Harvard community has made this article openly available.

Please share how this access benefits you. Submit a story.

Accessibility 


\title{
Default Mode Network Connectivity Encodes Clinical Pain: An Arterial Spin Labeling Study
}

\author{
Marco L. Loggia ${ }^{1,2,3,{ }^{*}, \text { Jieun Kim }}{ }^{3}$, Randy L. Gollub 2,3 , Mark G. Vangel ${ }^{3}$, Irving Kirsch ${ }^{4}$, Jian \\ Kong $^{2,3}$, Ajay D. Wasan ${ }^{1,5, \neq}$, and Vitaly Napadow ${ }^{1,3, \neq}$ \\ ${ }^{1}$ Department of Anesthesiology, Perioperative and Pain Medicine, Brigham and Women's \\ Hospital, Harvard Medical School (HMS), Boston, MA \\ ${ }^{2}$ Department of Psychiatry, Massachusetts General Hospital, HMS, Boston, MA \\ ${ }^{3} \mathrm{MGH} / \mathrm{MIT} / \mathrm{HMS}$ Athinoula A. Martinos Center for Biomedical Imaging, Charlestown, MA \\ ${ }^{4}$ Program in Placebo Studies \& Therapeutic Encounter, Beth Israel Deaconess Medical Center, \\ HMS, Boston MA, USA \\ ${ }^{5}$ Department of Psychiatry, Brigham and Women's Hospital, HMS, Boston, MA
}

\begin{abstract}
Neuroimaging studies have suggested the presence of alterations in the anatomo-functional properties of the brain of patients with chronic pain. However, investigation of the brain circuitry supporting the perception of clinical pain presents significant challenges, particularly when using traditional neuroimaging approaches. While potential neuroimaging markers for clinical pain have included resting brain connectivity, these cross-sectional studies have not examined sensitivity to within-subject exacerbation of pain. We used the dual regression probabilistic Independent Component Analysis approach to investigate resting-state connectivity on Arterial Spin Labeling (ASL) data. Brain connectivity was compared between patients with chronic low back pain (cLBP) and healthy controls, before and after the performance of maneuvers aimed at exacerbating clinical pain levels in the patients. Our analyses identified multiple resting state networks, including the Default Mode Network (DMN). At baseline, patients demonstrated stronger DMN connectivity to the pregenual anterior cingulate cortex (pgACC), left inferior parietal lobule and right insula (rINS). Patients' baseline clinical pain correlated positively with connectivity strength between the DMN and right insula (DMN-rINS). The performance of calibrated physical maneuvers induced changes in pain, which were paralleled by changes in DMN-rINS connectivity. Maneuvers also disrupted the DMN-pgACC connectivity, which at baseline was anti-correlated with pain. Finally, baseline DMN connectivity predicted maneuver-induced changes in both pain and DMN-rINS connectivity. Our results support the use of ASL to evaluate clinical pain, and the use of resting DMN connectivity as a potential neuroimaging biomarker for chronic pain perception.
\end{abstract}

(C) 2012 International Association for the Study of Pain. Published by Elsevier B.V. All rights reserved.

"Correspondence should be addressed to Dr. Marco L. Loggia, Massachusetts General Hospital, Building 75, 2132-A, Charlestown, MA 02129. marco@nmr.mgh.harvard.edu; Phone: +1 617-643-7267; Fax : +1-617-643-7340.

₹denotes equal contribution

The authors of the article declare that they have no competing financial interests.

Publisher's Disclaimer: This is a PDF file of an unedited manuscript that has been accepted for publication. As a service to our customers we are providing this early version of the manuscript. The manuscript will undergo copyediting, typesetting, and review of the resulting proof before it is published in its final citable form. Please note that during the production process errors may be discovered which could affect the content, and all legal disclaimers that apply to the journal pertain. 


\section{Keywords}

resting state networks; chronic low back pain; functional magnetic resonance imaging; psychophysics; human

\section{Introduction}

Neuroimaging studies have provided considerable evidence indicating that chronic pain is associated with structural, functional and neurochemical alterations distributed across multiple brain networks [50]. In spite of such progress, the identification of neural measures underlying the perception of clinical pain itself presents methodological hurdles. Unlike experimental pain (e.g., exogenous heat stimulus applied to the skin), clinical pain (e.g., endogenous pain in a patient suffering from low back pain) is difficult to elicit in a controlled manner. This fact makes it challenging to probe its neural correlates using classical 'two-state subtraction' (i.e., block- and event-related) neuroimaging designs [3]. Hence, alternative fMRI approaches have been adopted. For instance, our recent studies have reported an association between clinical pain intensity at the time of the scan and patterns of intrinsic brain connectivity [32; 33]. While the observation that brain activity or connectivity covaries with clinical pain is intriguing, correlational analyses alone, in the absence of any concomitant experimental manipulation, do not allow us to conclusively determine whether these patterns are specific to the perception of clinical pain. And thus, the current approaches limit an understanding of the mechanistic relationships between brain function and chronic pain perception. Specifically, while potential neuroimaging markers for clinical pain have included resting brain connectivity, its sensitivity to within-subject exacerbation of pain is unknown. In the present study, we assessed the effect of experimental exacerbation of clinical pain on connectivity of the Default Mode Network (DMN; $[11 ; 40]$. Our study builds on the growing evidence supporting altered brain processing within the DMN in chronic pain patients $[5-7 ; 14 ; 33 ; 49]$.

Patients with chronic low back pain (cLBP) and healthy controls were imaged with Arterial Spin Labeling (ASL) at rest (i.e., absent any stimulation during scanning), before and after a series of physical maneuvers aimed to exacerbate clinical pain in patients, but painless in controls [55]. While all patients received the same sequence of individually tailored maneuvers, the magnitude of change in clinical pain (from baseline) at the post-maneuver scan varied significantly across patients. As our recent studies have linked clinical pain intensity and resting DMN connectivity to insula [32;33], we hypothesized that withinsubject experimentally-induced changes in clinical pain would be associated with proportional changes in DMN-insula connectivity. Furthermore, the activity of DMN regions increases whenever a subject's attention is focused introspectively [11], is modulated by the behavioral relevance of a stimulus [18; 46], and has been found to predict behavior in a variety of tasks $[27 ; 29 ; 41]$. Therefore, we also hypothesized that baseline DMN connectivity would predict the amount of pain change reported by patients following maneuvers.

\section{Materials and Methods}

\section{General procedures}

We evaluated brain connectivity using resting Arterial Spin Labeling (ASL) data acquired from both cLBP patients and healthy controls in a previously published study [55]. In that study, ASL was the imaging technique of choice because in that study we wanted to quantify pain-induced regional cerebral blood flow (rCBF) changes in chronic low back pain patients. 
Full details of patients' characterization, stimulation protocol, scanning parameters and psychophysical results have been previously published [55]. All participants in the study provided written informed consent in accordance with the Human Research Committee of the Massachusetts General Hospital. Briefly, we studied 16 patients with chronic low back and radicular pain (mean $(\mathrm{CI})$ : age $=47.4$ years old $(40,54.8)$; pain duration $=6.24$ years $(3.9,11.8)$; baseline pain $(0-20)=6.4(2.8,5.9)$ Oswestry Disability Index score $=35.8(30$, 41.6), Pain Catastrophizing Scale score $=36(27.8,42.1), \%$ female $=69 ; \%$ with neuropathic pain $=44$ ), and 16 age- and gender-matched pain-free healthy controls (age $=$ $46.7(40.1,53.2), \%$ female $=69)$. None of the patients were being treated with opioid medications. Inclusion criteria for cLBP patients included a discogenic component to their pain, as determined by study physician (ADW), with the use of history, physical examination, and review of a lumbar MRI. All subjects participated in two imaging visits. During two imaging visits, two 6-minute pulsed ASL (pASL) scans were performed, before and after 12 clinical maneuvers ("clinical maneuvers" visit) or 12 heat pain stimuli ("heat pain" visit). The maneuvers (e.g., straight leg raise or pelvic tilt) were individually tailored in order to elicit a pain rating of $\sim 10-11$ ("moderate") or $\sim 14-15$ ("strong") on a 0-20 Numerical Rating Scale in patients, but were painless in the controls. Ratings were expressed on the Gracely Box Scale [23], which is a, ratio scale particularly suited and sensitive to determining the degree of change in pain within an experimental session.

For both patients and controls, heat pain stimuli were also individually tailored in order to elicit a "moderate" or a "strong" pain sensation. While the ASL data from the heat pain visit were included in the independent component analysis (see below) in order to provide a more solid estimation of the DMN, these were not included in any other step of the analysis because, unlike the clinical maneuvers session, the heat pain session A) did not produce a clinically significant increase in patients' pain $(19.4 \%$, vs. $34.3 \%$ in the clinical maneuvers visit [55]); and B) exhibited lower dynamic range in pain scores at baseline (0-9/20, vs. $1-15 / 20$ in the clinical maneuvers visit), thus limiting our inference power in all analyses.

\section{Imaging acquisition and analyses}

ASL timeseries were acquired on a 3 T Siemens TIM Trio MRI System (Siemens Medical, Erlangen, Germany), equipped with a 32-channel head coil, and using a PICORE-Q2TIPS sequence [31] TR/TE/TI1/TI2 $=3000 / 13 / 700 / 1700 \mathrm{~ms}$, voxel size $=3.515 * 3.515 * 6.25 \mathrm{~mm}$, number of slices $=16)$. A high resolution MPRAGE scan $(\mathrm{TR} / \mathrm{TE}=2300 / 3.39 \mathrm{~ms}$, voxel size $1 * 1 * 1.33 \mathrm{~mm}$ ) was also acquired in order to optimize spatial normalization to the MNI152 standard space.

ASL data preprocessing and analyses were performed using a combination of packages including FSL (FMRIB's Software Library, www.fmrib.ox.ac.uk/fsl) and Freesurfer (http:// surfer.nmr.mgh.harvard.edu/). The first tag-control pair was discarded to allow the MR signal to reach steady-state equilibrium. The remaining volumes were skull-stripped using BET and motion-corrected using MCFLIRT. Perfusion-weighted timeseries were obtained by pair-wise subtraction of adjacent tag and control images [1]. These timeseries were then registered to their respective Freesurfer-reconstructed high resolution anatomical volume using BBREGISTER [24], and then to the MNI152 standard space using FLIRT. The spatially normalized perfusion-weighted timeseries were finally highpass filtered (cutoff $=0.008 \mathrm{~Hz})$ and spatially smoothed $(\mathrm{FWHM}=5 \mathrm{~mm})$.

All the preprocessed ASL data obtained (for all subjects and for both visits) were concatenated to create a single 4D dataset. A probabilistic Independent Component Analysis (pICA; [8]) was performed using MELODIC on this concatenated 4D dataset in order to identify the resting state networks (RSNs). In order to select the parameter set that yielded the most reliable estimation of RSNs, this analysis was performed with different numbers of 
components $(25,40$ or 50$)$, using only the clinical maneuvers visit data or both visits' data, and with or without low-pass filtering. Using goodness-of-fit tests [17] with previously defined templates generously provided by Beckmann et al [8], we established that using 25 components on the low-pass filtered data from both imaging sessions yielded the most consistent RSN estimation. The subject-specific temporal dynamics and associated spatial maps of the DMN were calculated for both pre- and post-maneuver scans using the dual regression approach [21; 59]. In this technique, group-level spatial maps were used as a set of spatial regressors in a General Linear Model (GLM), in order to identify the individual subjects' timecourse associated with each group-level map. These timecourses were then variance normalized, and used as a set of temporal regressors in a GLM, to find subjectspecific maps associated with the different group-level independent components. In this GLM, explanatory variables also included timecourses from ventricles and white matter (but not global signal) as covariates of no interest. The dual regression technique is widely used, and has moderate-to-high test-retest reliability [59]. Subject-specific DMN maps were compared across groups as well as across timepoints (post-pre maneuvers) using unpaired and paired t-tests, respectively. We also evaluated the association between connectivity and pain intensity, as well as changes in both. For the former, we performed a regression analysis with baseline DMN connectivity and baseline pain as regressor of interest. For the latter, we first calculated DMN connectivity "change maps" (post-pre maneuvers) by subtracting parameter estimate maps for the pre-maneuvers scan from the maps corresponding to the post-maneuvers scan, for each subject. We also summed the associated variance images. These were entered into a regression model with regressor of interest being change in pain (post-pre maneuvers pain ratings). In order to test if significant clusters from this change-score regression were influenced by baseline connectivity or pain, we performed an ROI-based multiple linear regression analysis which allowed us to include both baseline pain and connectivity values as regressors of no interest. Finally, we evaluated the predictive capacity of baseline DMN connectivity, using a regression model with change in pain as regressor of interest, and baseline pain as regressor of no interest. Follow-up ROI-based multiple linear regression further allowed us to correct the imaging results for baseline pain, average pain rating reported during the maneuvers, and duration of pain. For ROI analyses, connectivity values (zstats) were averaged across all voxels from clusters of interest.

The group level analyses were performed using FLAME (FMRIB's Local Analysis of Mixed Effects) stage 1, with a voxel-wise cluster forming threshold of $Z=2.3$ and a (corrected) cluster significance threshold of $\mathrm{P}=0.05$. Finally, as the DMN-insula connectivity was found to be higher than in controls in patients with a different chronic pain condition (fibromyalgia) [33], and also to correlate with clinical pain in both that study as well as this one (see below), the baseline DMN connectivity maps were also compared across groups with a direct search restricted to the insula. This analysis was performed with an uncorrected threshold of $p<0.005$ and a minimum cluster size of 5 voxels, a procedure previously used in several imaging studies [39; 47].

\section{Results}

Following pICA on the concatenated ASL data, we were able to identify the majority of RSNs reported in previous BOLD fMRI resting state studies, including the default mode, medial and lateral visual, salience, right and left fronto-parietal control and dorsal attention networks (Figure 1). We confirmed that the component identified as the DMN by the goodness-of-fit tests included brain areas previously noted as 'core regions' of the DMN [12]: medial prefrontal cortex, posterior cingulate cortex / precuneus, inferior parietal lobule and lateral temporal cortex. 
Baseline (pre-maneuvers) DMN connectivity, assessed from resting ASL data using dual regression independent component analysis, was contrasted between patients and healthy controls (Figure 2; Tables 1,2). Whole-brain analyses revealed that, compared to controls, cLBP patients demonstrated stronger baseline DMN connectivity to the pregenual anterior cingulate cortex (pgACC), a component of the medial prefrontal cortex, as well as to the left inferior parietal lobule (Figure 2B, left). The strength of DMN-pgACC connectivity within this cluster was negatively correlated with clinical pain at baseline $(r=-0.73, p=0.001$; Figure 2B, right). Furthermore, a direct search also revealed a stronger DMN-insula connectivity in the patients (Figure 2B, bottom).

Experimental maneuvers, aimed at exacerbating clinical back pain in cLBP patients, on average significantly increased pain in these patients $(\mathrm{p}=0.005)$. Patients who reported clinical pain increase $(\mathrm{n}=13 ;=2.5 \pm 1.9$, on a scale of $0-20)$ exhibited a reduction in resting DMN connectivity to the medial prefrontal cortex, including pgACC, whereas controls, for whom the maneuvers were painless, instead demonstrated an increase. The interaction between TIME (post- vs. pre- maneuvers) and GROUP (cLBP vs controls) (Figure 2C, left) revealed a statistically significant cluster within a near identical region that demonstrated stronger DMN-pgACC connectivity at baseline (Figure 2B, left). An examination of the individual connectivity change scores (Figure $2 \mathrm{C}$, right) revealed that while no healthy controls demonstrated decreasing DMN-pgACC connectivity, cLBP patients demonstrated decrease in DMN-pgACC connectivity following physical maneuvers in all but three patients (empty circles). Intriguingly, these three patients were the only ones who actually reported a slight decrease, rather than an increase, in clinical pain at the end of the maneuvers (see below for more detail).

In order to determine whether DMN connectivity covaried with clinical pain intensity in cLBP patients in regions other than the pgACC, we performed a whole-brain regression analysis on the baseline DMN connectivity maps. The regressor of interest was clinical pain intensity. We found a single statistically significant cluster localized to the right mid-insula, extending slightly towards the adjacent putamen (Figure 3A; Table 3). As expected given the significant, negative relationship between DMN-pgACC connectivity and pain, baseline DMN-rINS connectivity was also negatively correlated with DMN-pgACC connectivity in patients $(\mathrm{r}=-0.60, \mathrm{p}=0.014)$. However, a partial correlation analysis between baseline pain, DMN-rINS, and DMN-pgACC connectivity revealed that when controlling for baseline pain, DMN-rINS and DMN-pgACC were no longer associated ( $\mathrm{r}=0.06, \mathrm{p}=0.83)$. This suggests that the correlation between these connectivity patterns is significantly associated with the presence of ongoing clinical pain. Indeed, resting DMN-rINS and DMN-pgACC were not correlated in pain-free controls $(\mathrm{r}=0.04, \mathrm{p}=0.896)$, further supporting this interpretation.

In order to assess whether within-session, maneuver-induced changes in mechanical low back pain were associated with changes in DMN connectivity, a second whole-brain regression analysis was performed on the within-session (post-pre maneuvers) DMN connectivity "change maps", using change score in clinical pain (post-pre maneuvers) as regressor. This analysis yielded another single cluster (Figure 3B; Table 3) on the right midinsula, overlapping (Figure 3C) with the insular component of the cluster observed in the baseline regression analysis. In order to ensure that the relationship between change in pain and change in connectivity was not driven by a significant correlation between baseline and change scores (suggesting, for example, a regression to the mean), we performed a followup ROI-based regression analysis using change in DMN-rINS connectivity as the dependent variable, change in pain as the independent variable, and including both baseline clinical pain and cluster connectivity as covariates of no interest. This analysis confirmed that, even 
after controlling for the strong relationship between baseline and change scores, changes in pain were still significantly $(\mathrm{p}=0.001)$ associated with changes in $\mathrm{DMN}$-insula connectivity.

In order to determine whether baseline DMN connectivity predicted changes in clinical pain, we performed another whole-brain regression analysis on the baseline DMN connectivity maps, using pain change scores (post-pre maneuvers) as regressor of interest, and baseline pain ratings as covariates of no interest. Indeed, baseline connectivity between DMN and posterior cingulate/retrosplenial cortex (PCC/RSp), right inferior parietal lobule (IPL) and left IPL extending into the lateral temporal cortex (LTC), predicted increasing pain following the maneuvers (Figure 4; Table 4). The predictive value of these baseline DMN connectivity patterns was statistically significant even when correcting for baseline pain, average pain rating reported during the maneuvers, and duration of pain in multiple regression analyses (PCC: $b=1.76, p=0.010 ;$ L IPL/LTC: $b=1.56, p<0.001 ;$ R IPL: $b=$ $2.36, \mathrm{p}<0.001)$. As expected given the abovementioned observation that DMN-insula connectivity encoded intensity of clinical pain, we further observed that baseline DMN connectivity within these regions also predicted maneuvers-induced changes in DMN-insula (although, after correcting for baseline DMN-insula connectivity, baseline pain and average pain reporting during the brief maneuvers only the R IPL results met strict criteria for statistical significance, $\mathrm{p}=0.026$; L IPL/LTC: $\mathrm{p}=0.09$; PCC: $\mathrm{p}=0.09$ ).

\section{Discussion}

In this study, DMN encoded the intensity of clinical pain, both at baseline and in response to maneuvers aimed at exacerbating clinical pain levels, and predicted post-maneuver lingering pain in cLBP patients. Our connectivity analyses used ASL fMRI data, and revealed that greater clinical pain at baseline was associated with greater DMN connectivity with the insula (a region known to be involved in pain processing [2]), and less connectivity with the pgACC (a region involved in pain inhibition $[9 ; 37 ; 54])$.

Notably, the DMN-rINS result was similar to that reported in our previous study investigating the association between clinical pain and DMN connectivity, but in a different chronic pain population (fibromyalgia) [33]. The observation that a common neuroimaging metric appears to encode clinical pain in different patient populations raises the intriguing possibility that such measures may reflect a general feature of chronic pain. This study, however, does not simply replicate previously published findings in a different patient population. In the present experiment, we did not limit ourselves to examine baseline correlations alone and implemented a paradigm with clinical pain exacerbation in order to test the sensitivity of such metrics to within-subject changes in clinical pain. This approach allowed us to observe that greater change in pain following physical maneuvers was associated with greater change in DMN-rINS. This result complements our observation that reduction in clinical pain induced by a 4-week non-pharmacological intervention in fibromyalgia patients was accompanied by a reduction in DMN-insula connectivity [32]. In sum, our study demonstrated that greater baseline clinical pain in cLBP patients was associated with greater DMN-rINS connectivity, and greater increase in mechanical low back pain following physical maneuvers was associated with greater increase in DMN-rINS connectivity. Altogether, these results support resting DMN-insula connectivity as a statespecific neuroimaging marker for clinical pain. Such association has now in fact been reproduced in different patient populations, and linked to within-subject increases and decreases in clinical pain, over both short and long time periods.

The fact that patients exhibited higher DMN-pgACC connectivity at baseline might reflect compensatory mechanisms taking place in preparation for the anticipated maneuver-induced pain increase. This compensatory mechanism would appear to be pain-protective, as the 
more pgACC connectivity at baseline, the lower the baseline clinical pain. While other subregions of the medial prefrontal cortex have been proposed to have a pain-facilitatory role [43], the pgACC has been extensively associated with anti-nociceptive functions [9;37; 54], likely exerted through its descending projections to the periaqueductal gray matter [53]. Our results further extend these observations by identifying a novel mechanism (pgACC connectivity with the DMN) through which pgACC might produce its pain-protective effects. Baliki and colleagues, in a seminal study, observed that DMN dynamics were disrupted in cLBP patients during the performance of a visual task [7], suggesting that this network is affected by a chronic pain state. Our study extends this finding, and establishes a direct linkage between DMN disruption and perceptual aspects of clinical pain in a chronic pain population.

Despite using individually calibrated maneuvers to elicit similar pain responses in all patients, the amount of change in clinical pain (compared to baseline) reported by patients after the stimulation was quite variable. As the post- vs pre-maneuvers change in pain was neither predicted by the baseline pain, nor by the average pain intensity reported during the brief maneuvers themselves, it appears that some patients were more susceptible to develop sustained clinical pain in response to a comparable stimulation paradigm. Since the activity of DMN regions is modulated by the behavioral relevance of a stimulus [18; 46], and has been found to predict behavior in a variety of tasks [27;29; 41], we hypothesized that baseline DMN connectivity would predict pain change following maneuvers. As such, we observed that baseline connectivity between $\mathrm{DMN}$ and posterior cingulate/retrosplenial cortex (PCC/RSp), right inferior parietal lobule (IPL) and left IPL/LTC predicted increasing pain following the maneuvers (Figure 4; Table 4).

Pre-stimulus brain activity/connectivity and sensitivity to stimuli or task performance have been the object of investigation in several studies $[4 ; 15 ; 22 ; 25 ; 48 ; 56]$. In the field of pain, recent studies on healthy volunteers receiving experimental pain stimuli have found that baseline activity of the anterior cingulate cortex and the insula predicted higher pain sensitivity in response to a subsequently presented experimental stimulus [10; 39], whereas the baseline activity of the periaqueductal gray and the functional connectivity between this midbrain region and the insula predicted lower pain sensitivity [39]. The present results significantly extend these findings, by providing evidence that a different neuroimaging metric, specifically resting DMN connectivity, can predict susceptibility to lingering clinical pain. Notably, our study on prediction of clinical pain did not identify regions common to the studies investigating prediction of experimental pain, possibly indicating that entirely different neurobiological underpinning may mediate hypersensitivity to experimental and clinical pain. While at this time the mechanisms by which increased DMN connectivity predicts stronger clinical pain exacerbations eludes a clear explanation, it is tempting to speculate that stronger connectivity within the DMN, a network which is thought to be involved in introspection and self-oriented cognition [11], might reflect hyperattention to clinical pain. Attentional focus, in fact, has been widely shown to affect pain perception [30; $42 ; 51]$ and hyperattention to pain has been discussed as a potential aberrant mechanism in chronic pain, including low back pain, patients [16]. Furthermore, the fact that DMN connectivity has also been implicated in negative affect [45] suggests that affective processing may also play a role in promoting lingering pain after the stimulation period.

ASL has been recently applied to evaluate pain processing [26; 34-36; 44; 55; 57]. While a handful of studies have recently demonstrated that connectivity analyses can be successfully employed on ASL data $[13 ; 19 ; 20 ; 38 ; 52 ; 58]$, to the best of our knowledge no such study has been published in the field of pain. Several features of the ASL technique confer potential advantages over BOLD [1], which might be beneficial in connectivity analyses. First, while the BOLD signal exhibits increasing noise at low frequencies, the power 
spectrum of the ASL signal is flat. This suggests that the estimation of DMN connectivity, which demonstrates peak power at relatively low frequencies $(0.008-0.1 \mathrm{~Hz})$, should be less affected by low frequency drifts, likely of non-neural origin, when estimation is made from ASL data. Second, whereas BOLD is dominated by venous signal, ASL is primarily sensitive to signal changes localized at the level of the capillary bed, and therefore evaluates activity more closely co-localized with neuronal and synaptic physiology. The unique possibility to perform functional connectivity analyses as well as to quantify regional cerebral blood flow from the same dataset demonstrates that ASL can be a very powerful and versatile technique for the investigation of brain correlates to chronic pain and other disorders of the central nervous system.

When comparing our ASL-based connectivity results with clinical pain-induced changes in rCBF previously reported [55], we noted that nearby MPFC and insula subregions were implicated in both analyses. For instance, pain-inducing maneuvers reduced DMN-MPFC connectivity and increased MPFC rCBF. While these results may seem at odds, they are not, as MPFC activation during clinical pain, a phenomenon also observed in other chronic pain populations [6; 43], might be the mechanism responsible for altered resting-state connectivity (an analogous interaction between functional connectivity and activation was observed by our laboratory for primary sensorimotor cortex [28]). While connectivity analyses revealed baseline differences between patients and controls, rCBF analyses did not [55]. Therefore, the observed group differences in connectivity appear to be at least partially independent from and complementary to the results of our rCBF analyses. Future studies will need to specifically dissect the mechanisms underlying the ostensibly complex relationship between connectivity changes and activation induced by pain.

Several limitations should be taken into consideration when interpreting the results from our study. First, the brain coverage of our ASL analyses did not include caudal structures, such as the brainstem and the cerebellum. As such, in the present experiment we could not investigate the activity of other regions that are crucial for pain processing and/or modulation. Future studies will be performed focusing on such regions. Furthermore, while every attempt was made to individually calibrate the clinical maneuvers, patients exhibited variability in their responses to the maneuvers. Although it could be argued that such variability might in part explain the differences in lingering pain observed at the end of the stimulation period, our results show that baseline DMN connectivity predicted lingering pain even after correcting for the pain reported after the maneuvers (as well as the baseline pain and pain duration).

In conclusion, we show that resting DMN connectivity encodes the severity of clinical pain, is sensitive to within-subject exacerbation of such pain, and can predict lingering clinical pain. In the future, studies will need to investigate the clinical implications of these observations, for instance by evaluating whether DMN connectivity can be used as a tool to predict which sub-clinical or acute pain patients go on to develop chronic pain.

\section{Acknowledgments}

We would like to thank Bruce Rosen, Randy Buckner and Karin Jensen for their helpful comments. We also thank Helen Chen and Amanda Cook for their help during the preparation of the manuscript. This study was supported in part by grants from NIH: K23-DA020681, R01-AT004714, P01-AT002048, R01-AT 005280, R01-AT006146, P01-AT006663.

\section{References}

1. Aguirre GK, Detre JA, Wang J. Perfusion fMRI for functional neuroimaging. Int Rev Neurobiol. 2005; 66:213-236. [PubMed: 16387205] 
2. Apkarian AV, Bushnell MC, Treede RD, Zubieta JK. Human brain mechanisms of pain perception and regulation in health and disease. Eur J Pain. 2005; 9(4):463-484. [PubMed: 15979027]

3. Apkarian AV, Krauss BR, Fredrickson BE, Szeverenyi NM. Imaging the pain of low back pain: functional magnetic resonance imaging in combination with monitoring subjective pain perception allows the study of clinical pain states. Neurosci Lett. 2001; 299(1-2):57-60. [PubMed: 11166937]

4. Arieli A, Sterkin A, Grinvald A, Aertsen A. Dynamics of ongoing activity: explanation of the large variability in evoked cortical responses. Science. 1996; 273(5283):1868-1871. [PubMed: 8791593]

5. Baliki MN, Baria AT, Apkarian AV. The cortical rhythms of chronic back pain. The Journal of neuroscience : the official journal of the Society for Neuroscience. 2011; 31(39):13981-13990. [PubMed: 21957259]

6. Baliki MN, Chialvo DR, Geha PY, Levy RM, Harden RN, Parrish TB, Apkarian AV. Chronic pain and the emotional brain: specific brain activity associated with spontaneous fluctuations of intensity of chronic back pain. The Journal of neuroscience : the official journal of the Society for Neuroscience. 2006; 26(47):12165-12173. [PubMed: 17122041]

7. Baliki MN, Geha PY, Apkarian AV, Chialvo DR. Beyond feeling: chronic pain hurts the brain, disrupting the default-mode network dynamics. The Journal of neuroscience : the official journal of the Society for Neuroscience. 2008; 28(6):1398-1403. [PubMed: 18256259]

8. Beckmann CF, DeLuca M, Devlin JT, Smith SM. Investigations into resting-state connectivity using independent component analysis. Philosophical transactions of the Royal Society of London Series B, Biological sciences. 2005; 360(1457):1001-1013.

9. Bingel U, Lorenz J, Schoell E, Weiller C, Büchel C. Mechanisms of placebo analgesia: rACC recruitment of a subcortical antinociceptive network. Pain. 2006; 120(1):8-15. [PubMed: 16364549]

10. Boly M, Balteau E, Schnakers C, Degueldre C, Moonen G, Luxen A, Phillips C, Peigneux P, Maquet $\mathrm{P}$, Laureys S. Baseline brain activity fluctuations predict somatosensory perception in humans. Proc Natl Acad Sci U S A. 2007; 104(29):12187-12192. [PubMed: 17616583]

11. Buckner RL, Andrews-Hanna JR, Schacter DL. The brain's default network: anatomy, function, and relevance to disease. Ann N Y Acad Sci. 2008; 1124:1-38. [PubMed: 18400922]

12. Buckner RL, Andrews-Hanna JR, Schacter DL. The brain's default network: anatomy, function, and relevance to disease. Ann N Y Acad Sci. 2008; 1124:1-38. [PubMed: 18400922]

13. Chuang KH, van Gelderen P, Merkle H, Bodurka J, Ikonomidou VN, Koretsky AP, Duyn JH, Talagala SL. Mapping resting-state functional connectivity using perfusion MRI. Neuroimage. 2008; 40(4):1595-1605. [PubMed: 18314354]

14. Cifre I, Sitges C, Fraiman D, Munoz MA, Balenzuela P, Gonzalez-Roldan A, Martinez-Jauand M, Birbaumer N, Chialvo DR, Montoya P. Disrupted Functional Connectivity of the Pain Network in Fibromyalgia. Psychosom Med. 2011

15. de Lafuente V, Romo R. Neuronal correlates of subjective sensory experience. Nat Neurosci. 2005; 8(12):1698-1703. [PubMed: 16286929]

16. Derbyshire SW, Jones AK, Creed F, Starz T, Meltzer CC, Townsend DW, Peterson AM, Firestone L. Cerebral responses to noxious thermal stimulation in chronic low back pain patients and normal controls. Neuroimage. 2002; 16(1):158-168. [PubMed: 11969326]

17. Dhond RP, Yeh C, Park K, Kettner N, Napadow V. Acupuncture modulates resting state connectivity in default and sensorimotor brain networks. Pain. 2008; 136(3):407-418. [PubMed: 18337009]

18. Downar J, Crawley AP, Mikulis DJ, Davis KD. The effect of task relevance on the cortical response to changes in visual and auditory stimuli: an event-related fMRI study. Neuroimage. 2001; 14(6):1256-1267. [PubMed: 11707082]

19. Farrell MJ, Bowala TK, Gavrilescu M, Phillips PA, McKinley MJ, McAllen RM, Denton DA, Egan GF. Cortical activation and lamina terminalis functional connectivity during thirst and drinking in humans. American journal of physiology Regulatory, integrative and comparative physiology. 2011; 301(3):R623-631.

20. Fernandez-Seara MA, Aznarez-Sanado M, Mengual E, Irigoyen J, Heukamp F, Pastor MA. Effects on resting cerebral blood flow and functional connectivity induced by metoclopramide: a perfusion MRI study in healthy volunteers. Br J Pharmacol. 2011; 163(8):1639-1652. [PubMed: 21175574] 
21. Filippini N, MacIntosh BJ, Hough MG, Goodwin GM, Frisoni GB, Smith SM, Matthews PM, Beckmann CF, Mackay CE. Distinct patterns of brain activity in young carriers of the APOEepsilon4 allele. Proc Natl Acad Sci U S A. 2009; 106(17):7209-7214. [PubMed: 19357304]

22. Fox MD, Snyder AZ, Vincent JL, Raichle ME. Intrinsic fluctuations within cortical systems account for intertrial variability in human behavior. Neuron. 2007; 56(1):171-184. [PubMed: 17920023]

23. Gracely RH, McGrath F, Dubner R. Ratio scales of sensory and affective verbal pain descriptors. Pain. 1978; 5(1):5-18. [PubMed: 673440]

24. Greve DN, Fischl B. Accurate and Robust Brain Image alignment using boundary-based registration. Neuroimage. 2009; 48:63-72. [PubMed: 19573611]

25. Hesselmann G, Kell CA, Eger E, Kleinschmidt A. Spontaneous local variations in ongoing neural activity bias perceptual decisions. Proc Natl Acad Sci U S A. 2008; 105(31):10984-10989. [PubMed: 18664576]

26. Howard MA, Krause K, Khawaja N, Massat N, Zelaya F, Schumann G, Huggins JP, Vennart W, Williams SC, Renton TF. Beyond patient reported pain: perfusion magnetic resonance imaging demonstrates reproducible cerebral representation of ongoing post-surgical pain. PLoS One. 2011; 6(2):e17096. [PubMed: 21373203]

27. Kim H. Neural activity that predicts subsequent memory and forgetting: a meta-analysis of 74 fMRI studies. Neuroimage. 2011; 54(3):2446-2461. [PubMed: 20869446]

28. Kim J, Loggia ML, Edwards RR, Wasan AD, LaCount L, Li A, Gollub RL, Harris RE, Park K, Napadow V. Functional brain connectivity is altered during prolonged experimental pressure pain. Hum Brain Mapp Abst. 2010

29. Li CS, Yan P, Bergquist KL, Sinha R. Greater activation of the "default" brain regions predicts stop signal errors. Neuroimage. 2007; 38(3):640-648. [PubMed: 17884586]

30. Loggia ML, Schweinhardt P, Villemure C, Bushnell MC. Effects of psychological state on pain perception in the dental environment. Journal. 2008; 74(7):651-656.

31. Luh WM, Wong EC, Bandettini PA, Hyde JS. QUIPSS II with Thin-Slice T11 Periodic Saturation: a Method for Improving Accuracy of Quantitative Perfusion Imaging USing Pulsed Arterial Spin Labeling. Magnetic Resonance in Medicine. 1999; 41:1246-1254. [PubMed: 10371458]

32. Napadow V, Kim J, Clauw DJ, Harris RE. Decreased intrinsic brain connectivity is associated with reduced clinical pain in fibromyalgia. Arthritis Rheum. 2012

33. Napadow V, LaCount L, Park K, As-Sanie S, Clauw DJ, Harris RE. Intrinsic brain connectivity in fibromyalgia is associated with chronic pain intensity. Arthritis Rheum. 2010; 62(8):2545-2555. [PubMed: 20506181]

34. Owen DG, Bureau Y, Thomas AW, Prato FS, St Lawrence KS. Quantification of pain-induced changes in cerebral blood flow by perfusion MRI. Pain. 2008; 136(1-2):85-96. [PubMed: 17716820]

35. Owen DG, Clarke CF, Bureau Y, Ganapathy S, Prato FS, St Lawrence KS. Measuring the neural response to continuous intramuscular infusion of hypertonic saline by perfusion MRI. Journal of magnetic resonance imaging : JMRI. 2012; 35(3):669-677. [PubMed: 21953816]

36. Owen DG, Clarke CF, Ganapathy S, Prato FS, St Lawrence KS. Using perfusion MRI to measure the dynamic changes in neural activation associated with tonic muscular pain. Pain. 2010; 148(3): 375-386. [PubMed: 19914778]

37. Petrovic P, Kalso E, Petersson KM, Ingvar M. Placebo and Opioid Analgesia-- Imaging a Shared Neuronal Network. Science. 2002; 295(5560):1737-1740. [PubMed: 11834781]

38. Pfefferbaum A, Chanraud S, Pitel AL, Muller-Oehring E, Shankaranarayanan A, Alsop DC, Rohlfing T, Sullivan EV. Cerebral blood flow in posterior cortical nodes of the default mode network decreases with task engagement but remains higher than in most brain regions. Cereb Cortex. 2011; 21(1):233-244. [PubMed: 20484322]

39. Ploner M, Lee MC, Wiech K, Bingel U, Tracey I. Prestimulus functional connectivity determines pain perception in humans. Proc Natl Acad Sci U S A. 2010; 107(1):355-360. [PubMed: 19948949]

40. Raichle ME, MacLeod AM, Snyder AZ, Powers WJ, Gusnard DA, Shulman GL. A default mode of brain function. Proc Natl Acad Sci U S A. 2001; 98(2):676-682. [PubMed: 11209064] 
41. Sala-Llonch R, Pena-Gomez C, Arenaza-Urquijo EM, Vidal-Pineiro D, Bargallo N, Junque C, Bartres-Faz D. Brain connectivity during resting state and subsequent working memory task predicts behavioural performance. Cortex; a journal devoted to the study of the nervous system and behavior. 2011

42. Schweinhardt P, Bushnell MC. Pain imaging in health and disease--how far have we come? The Journal of clinical investigation. 2010; 120(11):3788-3797. [PubMed: 21041961]

43. Schweinhardt P, Kalk N, Wartolowska K, Chessell I, Wordsworth P, Tracey I. Investigation into the neural correlates of emotional augmentation of clinical pain. Neuroimage. 2008; 40(2):759766. [PubMed: 18221890]

44. Segerdahl AR, Xie J, Paterson K, Ramirez JD, Tracey I, Bennett DL. Imaging the neural correlates of neuropathic pain and pleasurable relief associated with inherited erythromelalgia in a single subject with quantitative arterial spin labelling. Pain. 2012

45. Sheline YI, Price JL, Yan Z, Mintun MA. Resting-state functional MRI in depression unmasks increased connectivity between networks via the dorsal nexus. Proc Natl Acad Sci U S A. 2010; 107(24):11020-11025. [PubMed: 20534464]

46. Shulman GL, Astafiev SV, McAvoy MP, d'Avossa G, Corbetta M. Right TPJ deactivation during visual search: functional significance and support for a filter hypothesis. Cereb Cortex. 2007; 17(11):2625-2633. [PubMed: 17264254]

47. Singer T, Seymour B, O’Doherty JP, Stephan KE, Dolan RJ, Frith CD. Empathic neural responses are modulated by the perceived fairness of others. Nature. 2006; 439(7075):466-469. [PubMed: 16421576]

48. Super H, van der Togt C, Spekreijse H, Lamme VA. Internal state of monkey primary visual cortex (V1) predicts figure-ground perception. The Journal of neuroscience : the official journal of the Society for Neuroscience. 2003; 23(8):3407-3414. [PubMed: 12716948]

49. Tagliazucchi E, Balenzuela P, Fraiman D, Chialvo DR. Brain resting state is disrupted in chronic back pain patients. Neurosci Lett. 2010; 485(1):26-31. [PubMed: 20800649]

50. Tracey I, Bushnell MC. How neuroimaging studies have challenged us to rethink: is chronic pain a disease? J Pain. 2009; 10(11):1113-1120. [PubMed: 19878862]

51. Villemure C, Bushnell MC. Cognitive modulation of pain: how do attention and emotion influence pain processing? Pain. 2002; 95(3):195-199. [PubMed: 11839418]

52. Viviani R, Messina I, Walter M. Resting State Functional Connectivity in Perfusion Imaging: Correlation Maps with BOLD Connectivity and Resting State Perfusion. PLoS One. 2011; 6(11):e27050. [PubMed: 22073252]

53. Vogt, BA.; Sikes, RW.; Vogt, LJ. Anterior cingulate cortex and the medial pain system. In: Gabriel, M., editor. Neurobiology of cingulate cortex and limbic thalamus: a comprehensive handbook. Boston, MA: Birkhauser; 1993. p. 313-344.

54. Wager TD, Rilling JK, Smith EE, Sokolik A, Casey KL, Davidson RJ, Kosslyn SM, Rose RM, Cohen JD. Placebo-Induced Changes in fMRI in the Anticipation and Experience of Pain. Science. 2004; 303(5661):1162-1167. [PubMed: 14976306]

55. Wasan AD, Loggia ML, Chen LQ, Napadow V, Kong J, Gollub RL. Neural correlates of chronic low back pain measured by arterial spin labeling. Anesthesiology. 2011; 115(2):364-374. [PubMed: 21720241]

56. Weissman DH, Roberts KC, Visscher KM, Woldorff MG. The neural bases of momentary lapses in attention. Nat Neurosci. 2006; 9(7):971-978. [PubMed: 16767087]

57. Zeidan F, Martucci KT, Kraft RA, Gordon NS, McHaffie JG, Coghill RC. Brain mechanisms supporting the modulation of pain by mindfulness meditation. The Journal of neuroscience : the official journal of the Society for Neuroscience. 2011; 31(14):5540-5548. [PubMed: 21471390]

58. Zou Q, Wu CW, Stein EA, Zang Y, Yang Y. Static and dynamic characteristics of cerebral blood flow during the resting state. Neuroimage. 2009; 48(3):515-524. [PubMed: 19607928]

59. Zuo XN, Kelly C, Adelstein JS, Klein DF, Castellanos FX, Milham MP. Reliable intrinsic connectivity networks: test-retest evaluation using ICA and dual regression approach. Neuroimage. 2010; 49(3):2163-2177. [PubMed: 19896537] 


\section{A. DEFAULT MODE NETWORK}

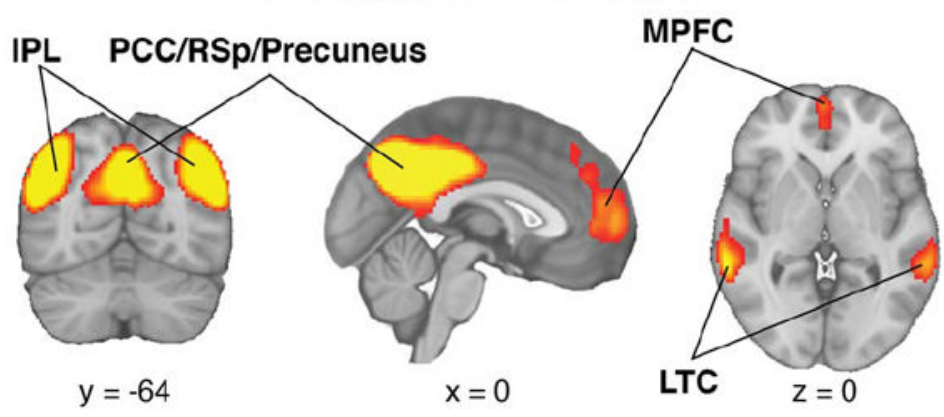

\section{B. OTHER RESTING STATE NETWORKS}

MEDIAL VISUAL

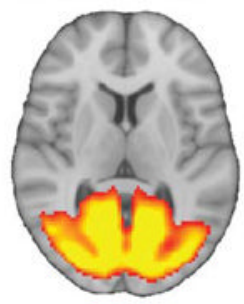

$z=+8$

R FRONTOPAR. CTRL

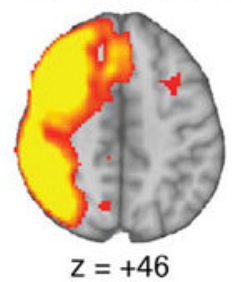

LATERAL VISUAL

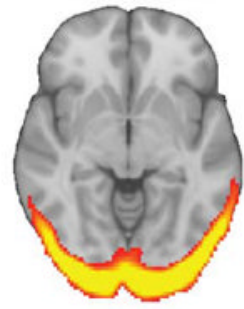

$z=-6$

L FRONTOPAR. CTRL

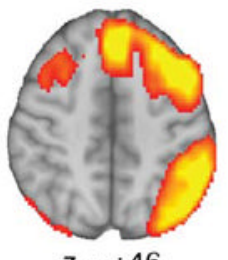

SALIENCE

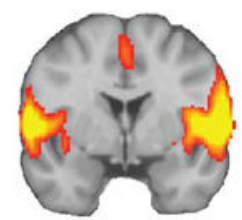

$y=0$

DORSAL ATTENTION

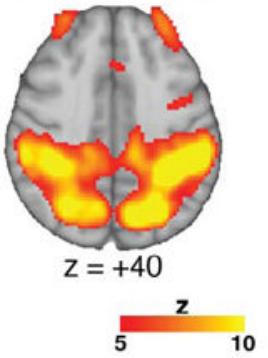

Figure 1. Resting-state networks identified using Arterial Spin Labeling

Probabilistic Independent component analysis on the ASL data allowed us to reliably identify several of the resting-state networks described in the BOLD fMRI literature, including the DMN (A) and other networks (B). IPL=Inferior Parietal Lobule; $\mathrm{PCC}=$ Posterior Cingulate Cortex; RSp=Retrosplenial Cortex, MPFC=Medial Prefrontal Cortex; LTC=Lateral Temporal Cortex; Frontopar. CTRL=Frontoparietal control 


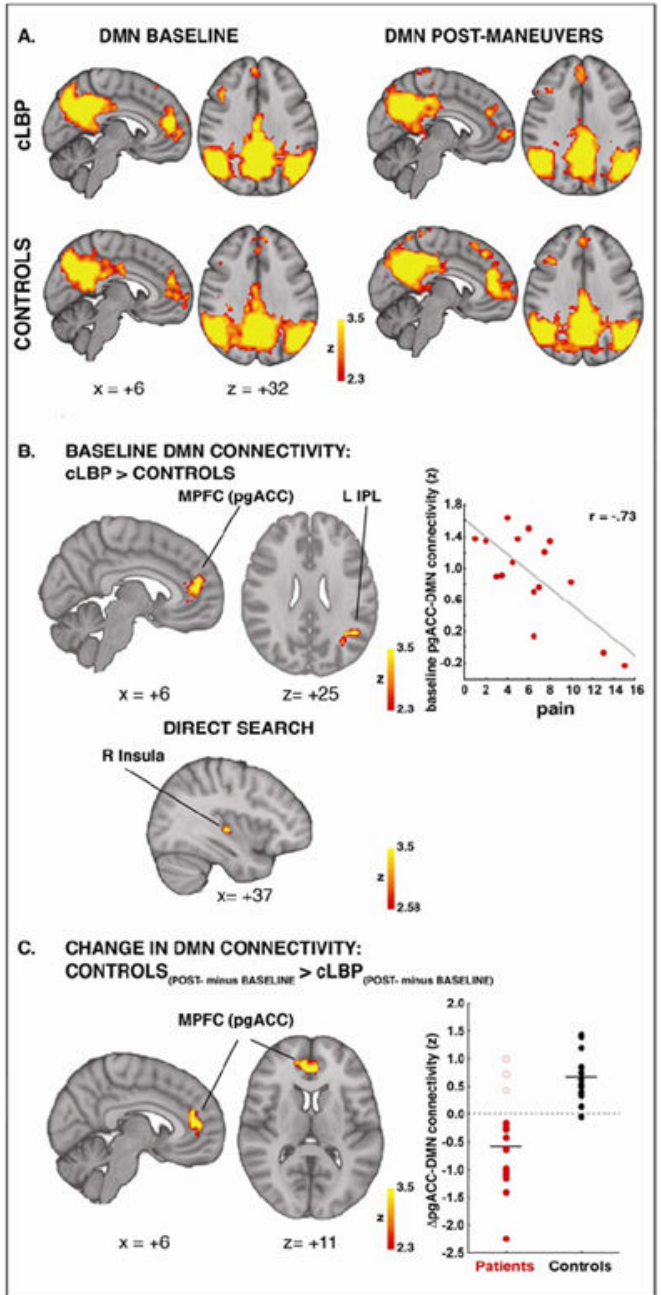

Figure 2. DMN connectivity is altered in cLBP patients

PgACC, L IPL and R insula are more connected to DMN in cLBP than in controls at baseline (A, left column; B). In patients, DMN-pgACC connectivity is negatively correlated with clinical pain at baseline $(\mathrm{B}$, right column) and is disrupted after the maneuvers (A, top row, C). After the maneuvers, all patients exhibited a reduction in DMN-pgACC connectivity, except for the three single patients (empty circles) who did not report an increase in pain. $\mathrm{DMN}=$ Default Mode Network; pgACC=pregenual anterior cingulate cortex. 


\section{A. DMN CONNECTIVITY vs PAIN AT BASELINE}
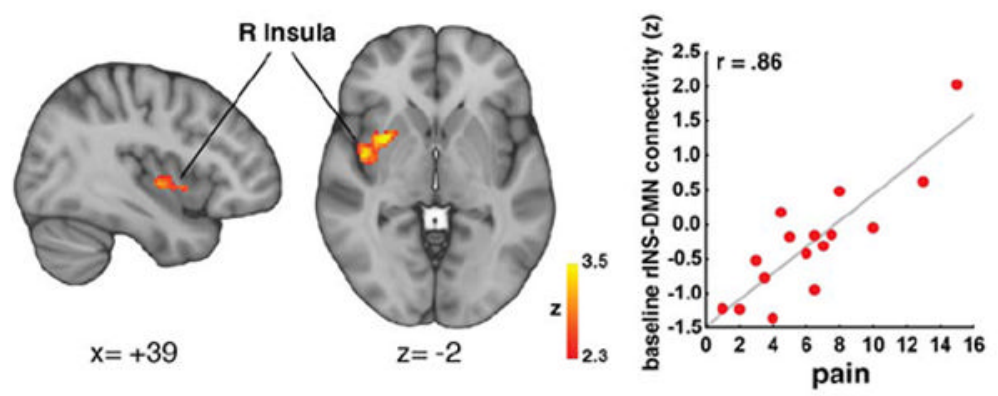

\section{B. $\triangle D M N$ CONNECTIVITY vs $\triangle$ PAIN (post-maneuvers minus baseline)}

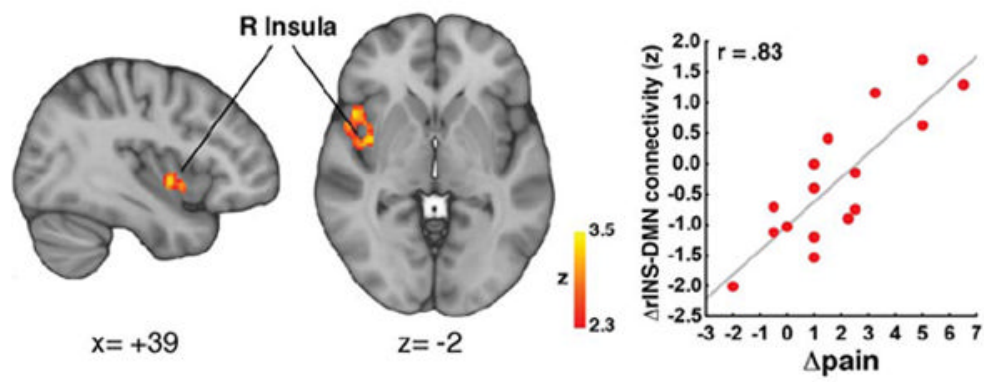

\section{CLUSTER INTERSECTION}

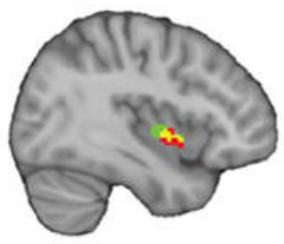

$x=+39$

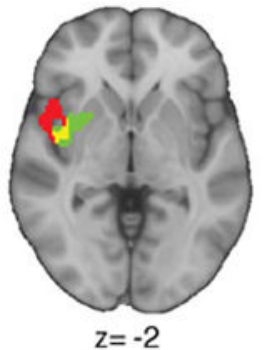

BASELINE

POST-MANEUV. MINUS BASELINE

INTERSECTION

Figure 3. Connectivity strength between right insula and DMN (DMN-rINS) correlates with clinical pain

Clinical pain correlated with DMN-rINS connectivity at baseline (A). Maneuvers-induced changes in pain correlated with changes in DMN-rINS connectivity (B). These analyses identified clusters overlapping over the right mid-insula (C). The $r$ values displayed in the scatterplots were computed from data extracted from significant clusters in the whole brain regression analyses, and are reported here for illustrative purposes. 


\section{BASELINE DMN CONNECTIVITY PREDICTS POST-MANEUVERS CHANGE IN PAIN AND DMN-rINS CONNECTIVITY}

A.
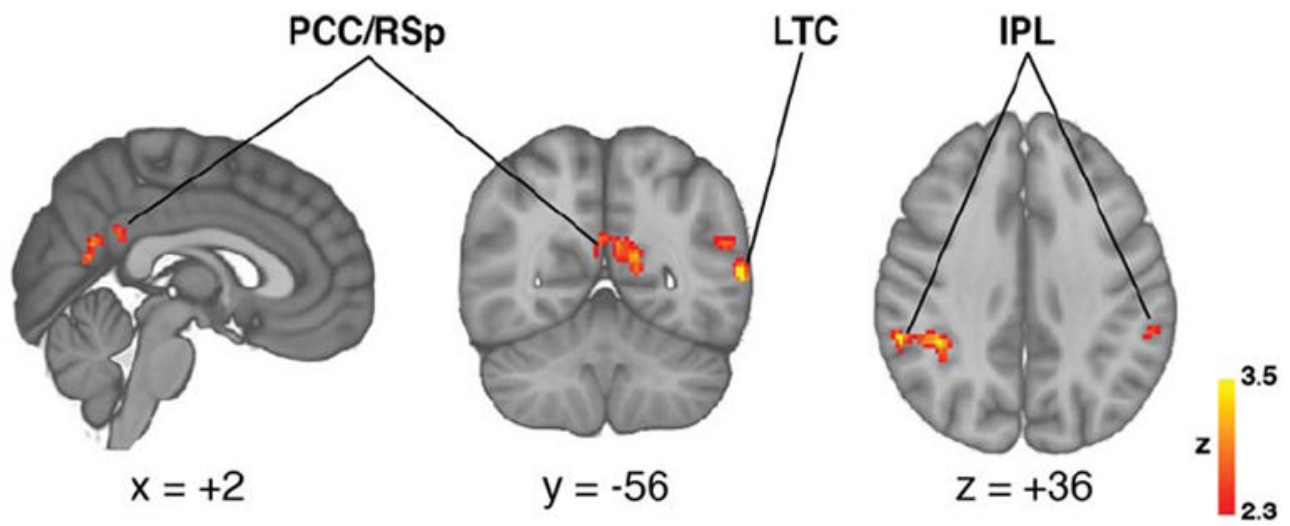

B.

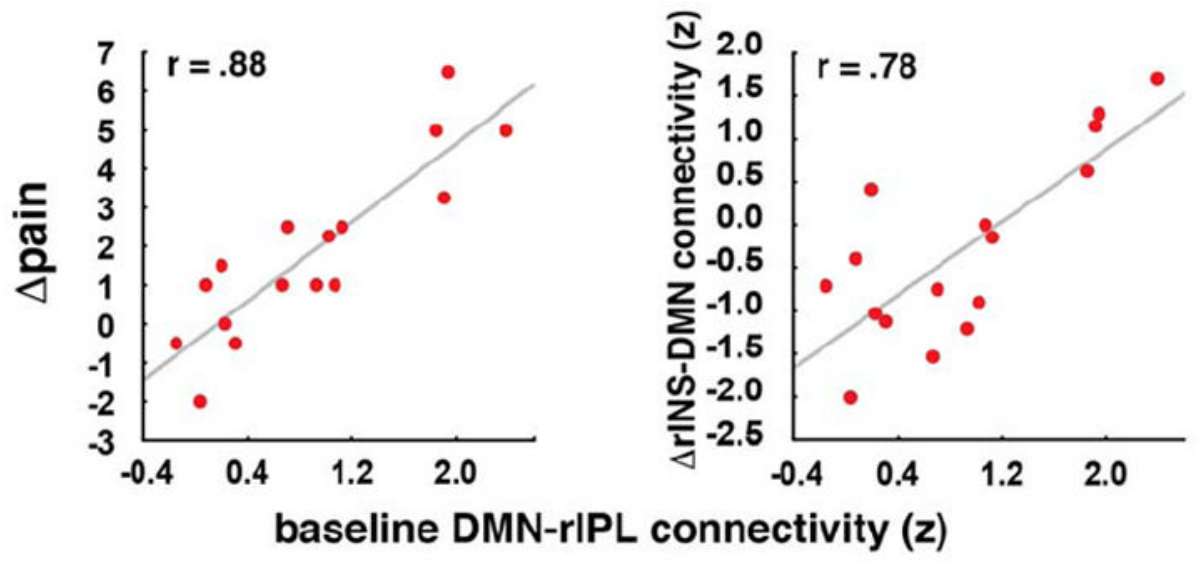

Figure 4. DMN baseline connectivity predicts sensitization to the clinical maneuvers Patients exhibiting the strongest connectivity between posterior regions of the DMN and the rest of the DMN reported the most severe pain increase after the maneuvers. IPL=Inferior Parietal Lobule; PCC=Posterior Cingulate Cortex; LTC=Lateral Temporal Cortex. 


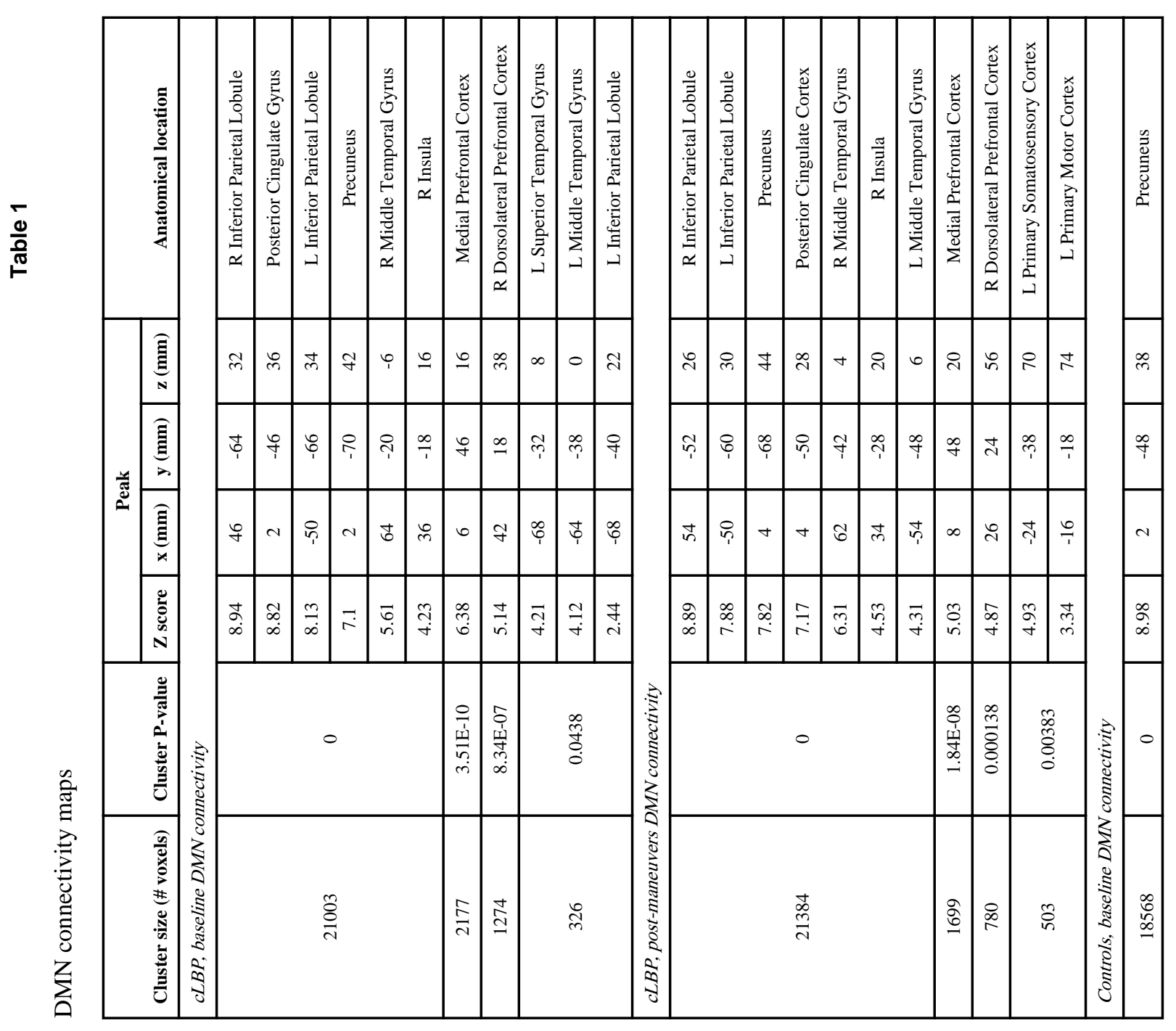

Pain. Author manuscript; available in PMC 2014 January 01. 


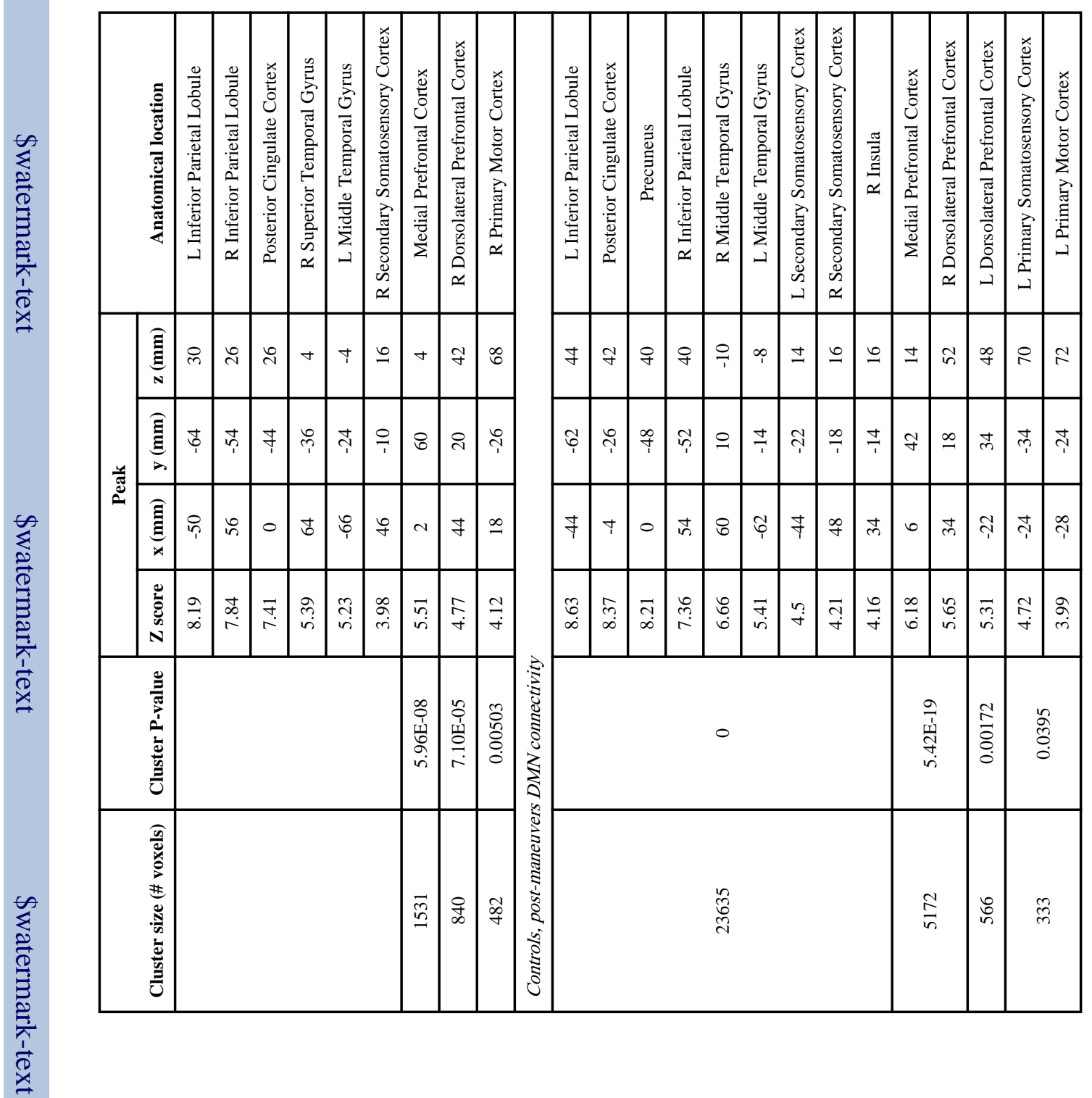




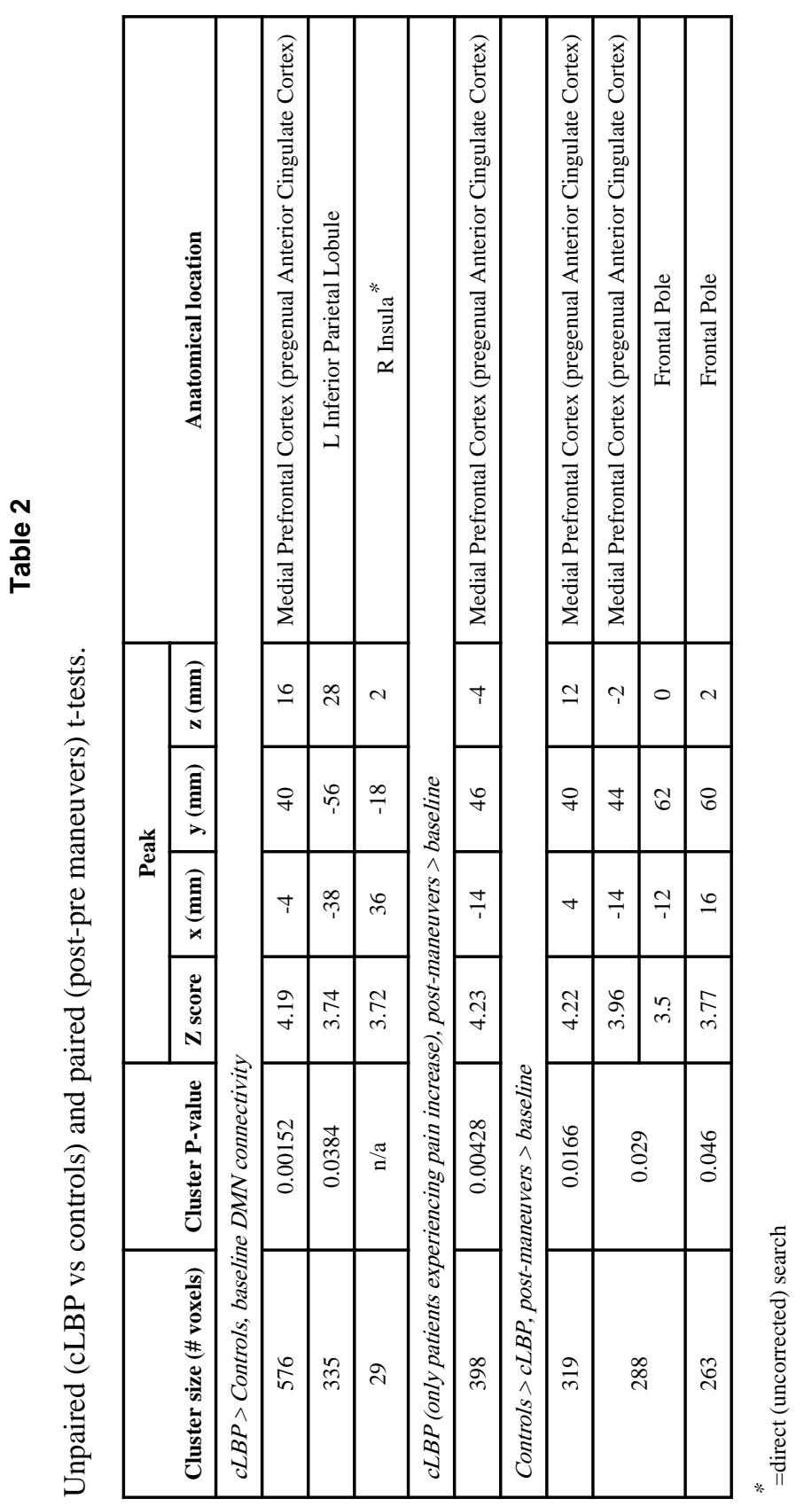

Pain. Author manuscript; available in PMC 2014 January 01. 


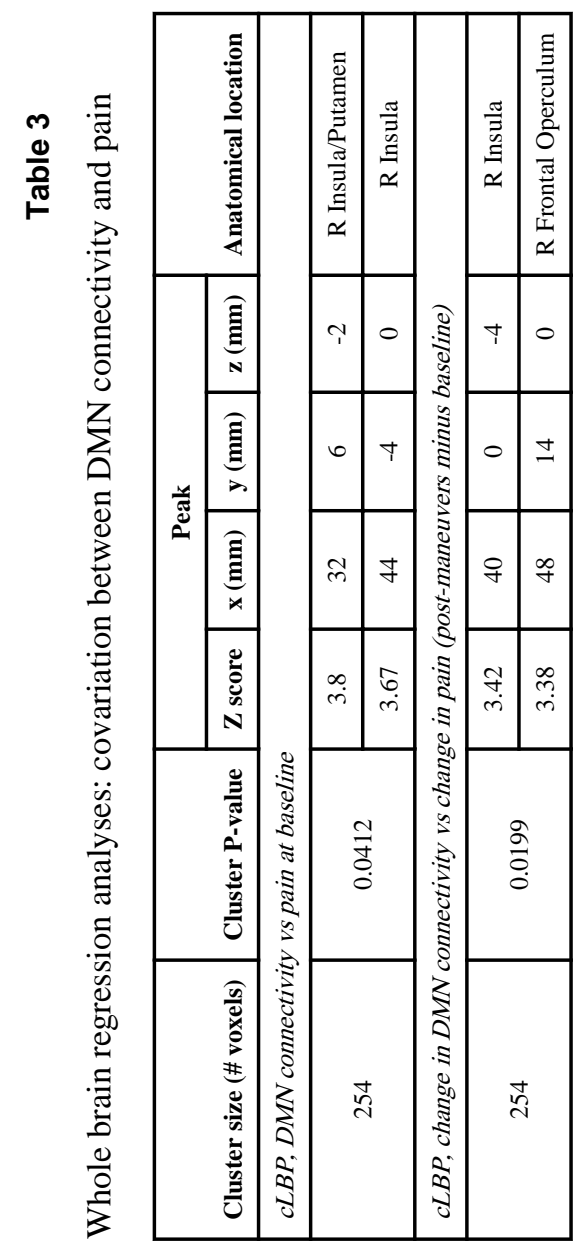

Pain. Author manuscript; available in PMC 2014 January 01. 


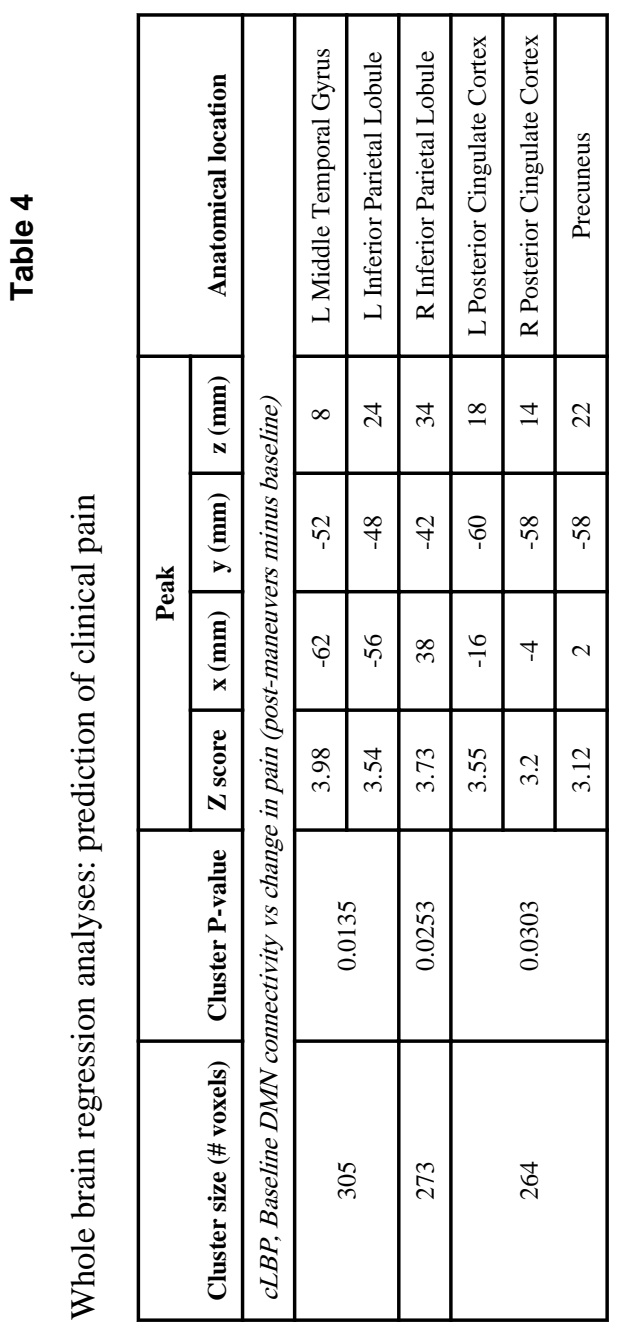

Pain. Author manuscript; available in PMC 2014 January 01. 